asthma when compared to non-severe asthma. Eosinophilic disease was significantly lower in the OSA group, suggesting an alternative driver of symptoms with this asthma phenotype. The current screening questionnaire failed to predict OSA in this specific population, suggesting routine overnight sleep studies are required to screen patients with severe asthma.

\section{P203 A COMPARISON OF ADVERSE EVENTS ASSOCIATED WITH LICENSED AND UNLICENSED SPACER USE WITH NON-EXTRAFINE BECLOMETASONE DIPROPIONATE TREATMENT IN A REAL-LIFE PATIENT POPULATION WITH ASTHMA IN THE UK}

${ }^{1} S$ Wan Yau Ming, ${ }^{2} \mathrm{~J}$ Haughney, ${ }^{3} \mathrm{D}$ Ryan, ${ }^{4} \mathrm{~S}$ Patel, ${ }^{4} \mathrm{M}$ Ochel, ${ }^{1} \mathrm{~S}$ Thornhill, ${ }^{1} \mathrm{D}$ Price. ${ }^{1}$ Observational and Pragmatic Research Institute, Singapore; ${ }^{2}$ University of Aberdeen, Aberdeen, UK; ${ }^{3}$ Optimum Patient Care, Cambridge, UK; ${ }^{4}$ Chiesi Ltd, Manchester, UK

\subsection{6/thoraxjnl-2017-210983.345}

Background Spacers may be compatible but unlicensed for an inhaler device. A prior study showed that co-prescription of unlicensed spacers with non-extrafine (non-EF) beclometasone dipropionate (BDP) asthma therapy was common. ${ }^{1}$ This study aimed to compare the occurrence of patient-reported inhaled corticosteroid (ICS)-related adverse events (AEs) in patients with asthma co-prescribed the licensed Volumatic or unlicensed Aerochamber spacer with their non-EF BDP therapy.

Method A cross-sectional study using completed questionnaires and data extracted from the Optimum Patient Care Research Database including patients with asthma, aged $\leq 65$ years, $\geq 2$ prescriptions for non-EF BDP and co-prescription of either a Volumatic or Aerochamber spacer. Patient characterisation was performed for the year prior to receipt of the completed questionnaire. AEs captured via questionnaire included continual sore mouth/throat, oral thrush, hoarse voice, bruising, weight gain and cough. The primary outcome was non-inferiority of the proportion of patient-reported oral candidiasis (reported as oral thrush/hoarse voice). The two spacer groups were compared using logistic regression, adjusted for gender, ICS average dose and smoking status. Non-inferiority was claimed if the upper $95 \%$ confidence interval (CI) of the marginal effect estimate was $<0.13$. Comparisons by age $(<16$, $16-65, \geq 65$ years $)$ and ICS dose $(<1000 \mu \mathrm{g}, \geq 1000 \mu \mathrm{g})$ were additionally performed.

Results Of the patients co-prescribed the licensed Volumatic spacer $(n=155), 29.9 \%$ reported oral candidiasis, compared to $27.7 \%$ of patients co-prescribed the unlicensed Aerochamber device $(n=385, p=0.622)$. The marginal effect estimate was $-0.043(95 \%$ CI $-0.133,0.047)$ and the Aerochamber was determined to be non-inferior to the Volumatic spacer. In terms of the total number of reported AEs, there were no significant differences for the main population ( $\leq 65$ years) $(p=0.797), 16-65$ years $(p=0.875),<16$ years $(p=0.687), \geq 65$ years $(p=0.425)$, high dose $(p=0.084)$ and low dose $(p=0.443)$ groups between those co-prescribed a Volumatic or an Aerochamber spacer.

Conclusion Co-prescription of the unlicensed Aerochamber spacer with non-EF BDP asthma therapy, in recommended patient groups, does not increase the risk of developing oral candidiasis or other ICS-related AEs, as compared to co-prescription of the licensed, Volumatic device.

\section{P204} PREVALENCE OF ANXIETY AND DEPRESSION IN PATIENTS WITH SEVERE ASTHMA

1J Finnerty, ${ }^{2} \mathrm{G}$ Paszek, ${ }^{1} \mathrm{~N}$ Sehgal. ${ }^{1}$ North Manchester General Hospital, Pennine Acute Hospitals NHS Trust, Manchester, UK; ${ }^{2}$ Greater Manchester Mental Health NHS Foundation Trust, Manchester, UK

\subsection{6/thoraxjnl-2017-210983.346}

Introduction Anxiety and depression correlate with poor asthma control. There is a cyclical relationship: poor asthma control increasing likelihood of emotional distress, which in turn reduces patient adherence and healthcare utilisation. In this study we assessed quality of life and the prevalence of depression and anxiety in patients with severe asthma.

Methods 55 patients on step 4-5 therapy attending the severe asthma clinic at North Manchester General Hospital, completed two questionnaires anonymously: Hospital Anxiety and Depression Scale (HADS) and Health Survey Short-Form (SF36). The HADS yields scores for anxiety and depression with scores greater than or equal to $8 / 21$ in either domain considered clinically significant. SF-36 scores were transformed into composite mental and physical component scores, with scores $<40$ considered poor.

Results $49 \%$ and $31 \%$ of the sample had clinically significant anxiety and depression respectively. 33\% had scores falling in the moderate-severe range for anxiety and/or depression with 5 patients having co-existing moderate-severe anxiety and depression. Patients reported poor quality of life relating to their physical (50\%) and mental health (33\%).

Conclusion The prevalence of anxiety in patients with severe asthma is significantly higher than in patients with chronic respiratory disorders $(49 \% \text { cf. } 29 \%)^{1}$ and is linked to poorer health and quality of life outcomes.

Extrapolating from our findings, we expect a third of patients in the asthma service $(\geq 500$ patients seen per year including 125 new referrals), to benefit from individual psychological support. NICE guidance recommends each should receive initial assessment and 12 therapy sessions (cognitive behavioural therapy/psychodynamic psychotherapies).

The position paper by the Kings Fund ${ }^{2}$ argued that mental health services should be delivered as part of an integrated approach to health. This study supports a clear role for an integrated physical health clinical psychology service as part of routine care for patients with severe asthma.

\section{REFERENCES}

1. Naylor, Haines, Vyas, Fowler. M3 anxiety and depression in patients with breath ing pattern disorders or chronic respiratory disease. Thorax 2015;70:A227-A228.

2. Naylor, Das, Ross, Honeyman, Thompson, Gilburt. (2016). Bringing together phys ical and mental health. A New Frontier for Integrated Care. London: The Kings Fund.

\section{Abstract P204 Table 1}

\begin{tabular}{lll}
\hline HADS $\mathrm{n}=55$ & Anxiety (HADS-A) & Depression (HADS-D) \\
\hline Non-case (0-7) & 28 & 38 \\
Mild (8-10) & 11 & 10 \\
Moderate (11-15) & 13 & 7 \\
Severe (16-21) & 3 & 0 \\
SF-36 $\mathrm{n=52*}$ & Physical component & Mental component \\
Non-case ( $\geq 40)$ & 26 & 35 \\
Poor quality of life range $(<40)$ & 26 & 17 \\
\hline *Incomplete data in 3 cases & &
\end{tabular}

